\title{
Exact results on the dynamics of multi-component Bose-Einstein condensate
}

\author{
Pijush K. Ghosh \\ Department of Physics, Ochanomizu University, \\ 2-1-1 Ohtsuka, Bunkyo-ku, Tokyo 112-8610, Japan.
}

\begin{abstract}
We study the time-evolution of the two dimensional multicomponent Bose-Einstein condensate in an external harmonic trap with arbitrary time-dependent frequency. We show analytically that the time-evolution of the total mean-square radius of the wave-packet is determined in terms of the same solvable equation as in the case of a single-component condensate. The dynamics of the total mean-square radius is also the same for the rotating as well as the non-rotating multi-component condensate. We determine the criteria for the collapse of the condensate at a finite time. Generalizing our previous work on a single-component condensate, we show explosion-implosion duality in the multi-component condensate.

PACS numbers: 03.75.Fi, 05.45.Yv, 11.15.-q, 03.65.Ge
\end{abstract}

The successful creation and observation of BoseEinstein condensation(BEC) in dilute alkali atoms have opened up a plethora of new possibilities to test, otherwise intractable, many-body quantum phenomenon in the laboratory [1]. The Gross-Pitaevskii equation(GPE), the mean-field description of the BEC, is successful enough in explaining most of the observed results as well as predicting new phenomenon. The methods involved in studying the GPE are mainly numerical and/or approximate: perturbative and variational. The exact and analytical results of a nonlinear equation, if known, not only act as a guide to determine the validity of different approximate and numerical methods; they also give rise to new, counter-intuitive results in some cases. Unfortunately, no exact solution of GPE is known except for in one dimension.

The two dimensional GPE, like its counterparts in higher dimensions, is not exactly solvable. However, due to an underlying dynamical $O(2,1)$ symmetry [2], the time-evolution of certain moments related to the two dimensional GPE can be described exactly [3]. This result is valid even if the condensate is considered in a timedependent harmonic trap. This leads to the prediction of explosion-implosion duality 䐗 and extended parametric resonance [4. 5] in the two dimensional BEC. Both of these phenomenon are universal for any non-relativistic theory having dynamical $O(2,1)$ symmetry [3, [, 1 ,6]. Interestingly enough, apart from the two dimensional BEC, the same explosion-implosion duality can also be observed in supernova explosion and in laser induced implosion in plasma [7.8. This shows the importance of exact methods, based on an underlying symmetry, in relating diverse areas of physics such as the BEC and the supernova ex- plosion.

The results described above are for a single-component condensate, where the spin degree's of freedom have been frozen though the use of a magnetic trap. Recently, the spinor condensate with independent spin degree's of freedom has also been created and observed in the laboratory [9]. Similarly, the two-component condensate, where two different hyperfine states of the same atomic species are condensed simultaneously, has also been experimentally realized [10]. The spinor condensate 11 14] and the two-component condensate [15] have a very rich structure compared to the single-component condensate. This is manifested in the existence of topological defects like skyrmion, domain-wall, vortices and Alice string in these condensates 16 .

The purpose of this note is to extend the studies of Refs. [3, A] on the two dimensional single-component BEC to the two dimensional multi-component BEC. The experimentally realizable two-component and the spinor condensate can be obtained as special cases of this general multi-component BEC. We study the exact time-evolution of the second moment of the two dimensional multi-component condensate in an arbitrary timedependent harmonic trap. This particular second moment can be identified as the total mean-square radius of the condensate. We show that the dynamics of the second moment is determined by the same solvable equation, as in the case of a single-component condensate. No matter how many components are there, or how they interact among themselves, or even whether they are rotating or non-rotating, the dynamics of the total mean-square radius is universally determined by the same equation. The detail information on the system is encoded, through the Hamiltonian, into a constant of motion appearing in this universal equation. Thus, the dynamics of the system can be studied in terms of the same set of initial conditions for any number of components. We determine the criteria for the collapse of the condensate of this system. We also show that the multi-component BEC, in its full generality, exhibits an explosion-implosion duality and extended parametric resonance for special choices of the time-dependence of the trap. All these results are exact and analytical.

Consider the following Lagrangian in $2+1$ dimensions,

$$
\begin{aligned}
\mathcal{L}= & \sum_{a}\left(i \psi_{a}^{*} \partial_{\tau} \psi_{a}-\frac{1}{2 m}\left|\nabla \psi_{a}\right|^{2}\right) \\
& -\frac{1}{2} \sum_{a b c d} g_{a b c d} \psi_{a}^{*} \psi_{b}^{*} \psi_{c} \psi_{d}, \quad a, b, c, d=1,2, \ldots, n,
\end{aligned}
$$


where $n$ is the total number of components. The coupling constants $g_{a b c d}$ are related to the s-wave scattering length matrix. The possible values of $g_{a b c d}$, and hence of the scattering length matrix, may be constrained by symmetry requirements. For example, the special case of a two-component condensate can be obtained by choosing $n=2$ and $g_{a b c d}=\frac{1}{2}\left(\delta_{a c} \delta_{b d}+\delta_{a d} \delta_{b c}\right) \bar{g}_{a b}$ so that the system has a global $U(1)^{2}$ symmetry. A phase-separation occurs for such a system if all the scattering lengths are positive and satisfy the inequality, $g_{12}^{2}=g_{21}^{2}>g_{11} g_{22}$ 15. Similarly, the spin one spinor condensate can be obtained by choosing $n=3$ and $g_{a b c d}=\frac{1}{2}\left[g_{1} \delta_{a c} \delta_{b d}+g_{2} \sum_{\alpha}\left(S_{\alpha}\right)_{a c}\left(S_{\alpha}\right)_{b d}+(a \leftrightarrow b)\right]$, where $S_{\alpha}$ 's are three spin-matrices. A positive $g_{2}$ defines an anti-ferromagnetic regime, while the ferromagnetic regime is characterized by a negative $g_{2}$. It is known that the ferromagnetic or the anti-ferromagnetic nature of the interaction plays an important role to characterize different properties of the condensate [11,12]. Both the phenomenon of the phase separation and the ferromagnetic or anti-ferromagnetic nature of the ground state are specific to multi-component condensate for $n \geq 2$. Further, note that we have additional terms describing the interaction among different components as we go from the single-component to the two-component, to spinor and to the general multi-component condensate described by (11). However, to our surprise, the dynamics of the total mean-square radius is independent of such variation in the inter-component interaction and universally determined by the same solvable equation as in the case of a single-component $(n=1)$ condensate. Consequently, the criteria for the collapse of the condensate at a finite time is also the same for any $n$-component condensate. For the very special case of an additional global $U(1)^{n}$ symmetry in (11), such a result has been obtained previously in Ref. [17]. We remark that our results are much more general. Moreover, the known results are reproduced in a very elegant way. We will consider only the most general form of $\mathcal{L}$ from now onwards, since our result is independent of particular details of the interaction.

All the coupling constants $g_{a b c d}$ have the inverse-mass dimension in the natural units with $c=\bar{h}=1$. This allows to have a scale and conformally invariant theory. The action $\mathcal{A}=\int d \tau d^{2} \mathbf{r} \mathcal{L}$ is invariant under the following time-dependent transformations 18 24,

$\mathbf{r} \rightarrow \mathbf{r}_{\mathbf{h}}=\dot{\tau}(t)^{-\frac{1}{2}} \mathbf{r}, \quad \tau \rightarrow t=t(\tau), \quad \dot{\tau}(t)=\frac{d \tau(t)}{d t}$
$\psi_{a}(\tau, \mathbf{r}) \rightarrow \psi_{a}^{h}\left(t, \mathbf{r}_{h}\right)=\dot{\tau}^{\frac{d}{4}} \exp \left(-i m \frac{\ddot{\tau}}{4 \dot{\tau}} r_{h}^{2}\right) \psi_{a}(\tau, \mathbf{r})$

with the scale-factor $\tau$ given by,

$$
\tau(t)=\frac{\alpha t+\beta}{\gamma t+\delta}, \quad \alpha \delta-\beta \gamma=1
$$

Note that all the components of the order parameter are multiplied by the same time-dependent scale-factor and the phase in the symmetry-transformation above. One might naively think that the requirement of the identical phase-factors for all the components of the order parameter is due to the interaction term. However, this is not the case. Even if we consider the free theory, i.e. $g_{a b c d}=0$ for all values of the indices, the requirement of the identical phases in (2) is essential in order it to be a symmetry transformation. This is precisely because the transformation of the scalar-fields is coupled with that of the space-time coordinates. If we choose some special values for the coupling constants $g_{a b c d}$ such that the Lagrangian has an internal global symmetry, say for example $S U(n)$, we certainly have the freedom of varying the phase-factors up to a global $S U(n)$ rotation. However, such an additional internal symmetry are completely decoupled from the symmetry transformations described in Eqs. (2) and (3), and do not have any effect on our results.

Let us now introduce two moments $I_{1}$ and $I_{2}$ in terms of the density $\rho$ and the current $\mathbf{j}$ as,

$$
\begin{aligned}
& \rho(\tau, \mathbf{r})=\sum_{a} \psi_{a}^{*} \psi_{a}, \\
& \mathbf{j}(\tau, \mathbf{r})=-\frac{i}{2 m} \sum_{a}\left(\psi_{a}^{*} \nabla \psi_{a}-\psi_{a} \boldsymbol{\nabla} \psi_{a}^{*}\right), \\
& I_{1}(\tau)=\frac{m}{2} \int d^{2} \mathbf{r} r^{2} \rho, \\
& I_{2}(\tau)=\frac{m}{2} \int d^{2} \mathbf{r} \mathbf{r} \cdot \mathbf{j} .
\end{aligned}
$$

We are dealing with a conservative system and the total number of particles $N(\tau)=\int d^{2} \mathbf{r} \rho$ is a constant of motion. The global $U(1)$ symmetry of $\mathcal{L}$ can be enlarged to $U(1)^{n}$ for certain special choices of $g_{a b c d}$. The total number of particles for each species are conserved separately for this case. However, as emphasized earlier, such an additional internal symmetry do not have any significant effect on our results. Thus, only the conservation of the total number of particles $N$ is important for our study. The moment $I_{1}$ is the sum of the mean-square radii corresponding to each and every components. This moment can be interpreted as the square of the width of the wave-packet for the single-component condensate, when confined in an external harmonic trap [5]. However, for the multi-component case, the moment $I_{1}$ can not be identified as the total width of the wave-packet. As emphasized in our previous work [3], the moment $I_{1}$ has been used extensively in the analysis of the non-linear Schrödinger equation( NLSE) [5,6,25 27], BEC [28] and in optics [29]. The dynamics of $I_{1}$, when the system (11) is immersed in an external time-dependent harmonic trap, is the central subject of the investigation of this letter. We show that the dynamics of $I_{1}$ is universally determined by the same solvable equation, as in the case of a single-component BEC. 
Particular choices of $\tau(t)=t+\beta, \alpha^{2} t$, and $\frac{t}{1+\gamma t}$, correspond to time translation, dilation and special conformal transformation. The corresponding generators of these transformations, the Hamiltonian $H$, the dilatation generator $D$ and the conformal generator $K$ are,

$$
\begin{aligned}
H & =\int d^{2} \mathbf{r}\left[\frac{1}{2 m} \sum_{a}\left|\nabla \psi_{a}\right|^{2}+\frac{1}{2} \sum_{a b c d} g_{a b c d} \psi_{a}^{*} \psi_{b}^{*} \psi_{c} \psi_{d}\right] \\
D & =\tau H-I_{2} \\
K & =-\tau^{2} H+2 \tau D+I_{1} .
\end{aligned}
$$

These generators close under the algebra,

$$
[H, D]=i H, \quad[H, K]=2 i D, \quad[K, D]=-i K,
$$

if we promote the fields $\psi_{a}$ 's to the operators $\hat{\psi}_{a}$ with the following bosonic commutation relations among themselves,

$$
\begin{aligned}
& {\left[\hat{\psi}_{a}(\mathbf{r}), \hat{\psi}_{b}^{*}\left(\mathbf{r}^{\prime}\right)\right]=\delta_{a b} \delta\left(\mathbf{r}-\mathbf{r}^{\prime}\right),} \\
& {\left[\hat{\psi}_{a}(\mathbf{r}), \hat{\psi}_{b}\left(\mathbf{r}^{\prime}\right)\right]=\left[\hat{\psi}_{a}^{*}(\mathbf{r}), \hat{\psi}_{b}^{*}\left(\mathbf{r}^{\prime}\right)\right]=0 .}
\end{aligned}
$$

The algebra given by Eq. (6) defines a conformal group, which is isomorphic to the group $O(2,1)$ 18. Thus, the system (11) has a dynamical $O(2,1)$ symmetry with the interpretation of the fields $\psi_{a}$ 's as the operators $\hat{\psi}_{a}$ satisfying (7). In this note, we will be considering only the fields $\psi_{a}$ 's, not the operators $\hat{\psi}_{a}$. We do not make use of the relations (7) or the algebra given by Eq. (6) in our subsequent discussions; what is required for our study is the conserved Noether charges $H, D$ and $K$. We just mention, in passing, that the results described in this note are valid for any non-relativistic theory with a dynamical $O(2,1)$ symmetry.

The generators $H, D$ and $K$ are constant in time and lead to the following equations,

$$
\frac{d H}{d \tau}=0, \quad \frac{d I_{1}}{d \tau}=2 I_{2}, \quad \frac{d I_{2}}{d \tau}=H
$$

For time independent solutions, both $I_{1}$ and $I_{2}$ do not depend on $\tau$. As a consequence, the energy of the static solutions of $H$ vanishes. This is also the case for the single-component BEC in $2+1$ dimensions. Even though there are extra terms due to inter-component interaction in the case of multi-component BEC, the vanishing of the energy is a universal consequence of the underlying $O(2,1)$ symmetry. The second equation of (8) shows that the moment $I_{2}$ is proportional to the time-variation of the moment $I_{1}$. Recalling that the moment $I_{1}$ is identified as the total mean-square radius of the condensate, the moment $I_{2}$ can be related to the speed of the growth of the condensate. This interpretation is also evident in the definition of $I_{2}$ in Eq. (4i) after decomposing the current $\mathbf{j}$ as a product of the density $\rho$ and the velocity.
Defining $X=\sqrt{I_{1}}$, it is easy to find a decoupled equation for $X$ from (8),

$$
\frac{d^{2} X}{d \tau^{2}}=\frac{Q}{X^{3}}, \quad Q=I_{1} H-I_{2}^{2}, \quad \frac{d Q}{d \tau}=0 .
$$

The constant of motion $Q$ is the Casimir operator of the $O(2,1)$ symmetry. Note that the information on the Hamiltonian $H$ is solely contained in $Q$. Thus, the effect of the interaction, say for example the strongly repulsive or attractive inter-component and intra-component interaction, will be manifested through initial conditions on $H$. Eq. (9) can be interpreted as the equation of motion of a particle moving in an inverse-square potential. Interestingly enough, this system also has a dynamical $O(2,1)$ symmetry. This reduced system of a particle in an inverse-square potential is a well-studied problem and the solution is given by 18,

$$
X^{2}=(a+b \tau)^{2}+\frac{Q}{a^{2}} \tau^{2},
$$

where $a$ and $b$ are integration constants. Although any exact solution of the equation of motion of the action $\mathcal{A}$ is not known, it is surprising to note how the exact timedependence of the moment $I_{1}$ can be obtained easily using the underlying symmetry. We would like to stress that we are able to determine the dynamics of the total mean-square radius of the condensate only. The dynamics of the individual mean-square radii associated with each components can not be obtained using our method even when there is an additional $U(1)^{n}$ symmetry in the system or there is no inter-component interaction.

The criteria for the collapse of the condensate at a finite and real time $\tau^{*}$ is $Q \leq 0$. In particular, the moment $X^{2}$ vanishes at a finite time $\tau^{*}$,

$$
\tau^{*}=\frac{a^{2}}{\left(a^{2} b^{2}+Q\right)}[-a b \pm \sqrt{-Q}],
$$

which is real if $Q \leq 0$. Note that we have the freedom of making $\tau^{*}$ either positive or negative by choosing appropriate values for the integration constants $a$ and $b$. With the interpretation of (9) as a particle moving in an inverse-square potential, the collapse of the condensate can be understood as the fall of the particle to the center for attractive interaction. Recall that the moment $I_{1}$ is semi-positive definite by definition. Thus, the exact expression for $Q$ implies that the condensate collapses for any initial condition if $H \leq 0$. On the other hand, if $H>0$, the condition for the collapse is given by,

$$
\left.\frac{d I_{1}}{d \tau}\right|_{\tau=0} \leq-2 \sqrt{\left.I_{1}\right|_{\tau=0}|H|} .
$$

We have used the second equation of (8) in the exact expression for $Q$ in deriving the above equation. As far as we are aware of, this is the first instance in 
the literature where the criteria for the collapse of the condensate of the most general two-dimensional multicomponent NLSE with cubic nonlinearity is given. The criteria is independent of the total number of components $n$ and any additional global internal symmetry. Thus, the well-known results on the single-component [25] and the multi-component [17] NLSE in two dimensions are easily reproduced from our general result.

Consider the following time-dependent transformation,

$$
\begin{aligned}
& \tau \rightarrow t=t(\tau), \quad \dot{\tau}(t)=\frac{d \tau(t)}{d t}, \\
& \mathbf{r} \rightarrow \mathbf{r}_{\mathbf{h}}=\dot{\tau}(t)^{-\frac{1}{2}} \quad\left(\begin{array}{cc}
\cos f(t) & \sin f(t) \\
-\operatorname{sinf}(t) & \cos f(t)
\end{array}\right) \mathbf{r} \\
& \psi_{a}(\tau, \mathbf{r}) \rightarrow \psi_{a}^{h}\left(t, \mathbf{r}_{h}\right)=\dot{\tau}^{\frac{d}{4}} \exp \left(-i m \frac{\ddot{\tau}}{4 \dot{\tau}} r_{h}^{2}\right) \psi_{a}(\tau, \mathbf{r}),
\end{aligned}
$$

with arbitrary $\tau(t)$ and $f(t)$. Note that this transformation can be obtained by first using the transformation (2) and then a time-dependent rotation around the z-axis with a time-dependent angle $f(t)$. For arbitrary $\tau(t)$ and $f(t)$, the transformation (13) is not a symmetry transformation of the action $\mathcal{A}$, instead, it maps $\mathcal{A}$ to a new action $\mathcal{A}_{h}=\int d t d^{2} \mathbf{r} \mathcal{L}_{h}$. The new Lagrangian $\mathcal{L}_{h}$ reads as,

$$
\begin{aligned}
\mathcal{L}_{h}= & \sum_{a}\left(i \psi_{a}^{h^{*}} \partial_{t} \psi_{a}^{h}-\frac{1}{2 m}\left|\nabla_{h} \psi_{a}^{h}\right|^{2}\right) \\
& -\frac{1}{2} \sum_{a b c d} g_{a b c d} \psi_{a}^{h^{*}} \psi_{b}^{h^{*}} \psi_{c}^{h} \psi_{d}^{h} \\
& -\sum_{a}\left(\frac{1}{2} m \omega(t) r_{h}^{2}\left|\psi_{a}^{h}\right|^{2}+\dot{f} \psi_{a}^{h^{*}} L_{z} \psi_{a}^{h}\right),
\end{aligned}
$$

where the z-component of the angular momentum, $L_{z}=$ $-i \mathbf{r}_{h} \times \nabla_{h}$ and the time-dependent frequency $\omega(t)$ of the harmonic trap is determined as,

$$
\ddot{b}+\omega(t) b=0, \quad b(t)=\dot{\tau}^{-\frac{1}{2}} .
$$

The Lagrangian $\mathcal{L}_{h}$ is that of a rotating multi-component BEC in an arbitrary time-dependent harmonic well. Note that the external harmonic potentials are identical for all the components of the condensate. This is not by a choice. In fact, we do not have the freedom of generating different harmonic potentials for different components using the transformation in Eq. (13). This is even true for the free theory, i.e. $g_{a b c d}=0$. The reason is that the transformation of the scalar fields is coupled with that of the space-time coordinates. Consequently, unphysical and unwanted terms will be generated in the new Lagrangian $\mathcal{L}_{h}$ unless all the components of the condensate transform identically.

The solutions of $\mathcal{A}$ and $\mathcal{A}_{h}$ are related to each other through the transformations in Eq. (13) with $\tau(t)$ determined for a specific trap-frequency by the Eq. (15). The scale-factor $\tau(t)$ can obviously be exactly determined for a large class of $\omega(t)$. However, the exact solutions are not known for either $\mathcal{A}$ or $\mathcal{A}_{h}$. This is a major problem in making use of the mapping relating $\mathcal{A}$ to $\mathcal{A}_{h}$ and the vice versa. However, note that the dynamics of the moment $I_{1}$ is uniquely determined by Eq. (10) independent of whether any exact solution of $\mathcal{A}$ is known or not. Thus, the transformation (13) can be used to find the dynamics of the moment $I_{1, h}=\sum_{a} \int d^{2} \mathbf{r}_{h} r_{h}^{2}\left|\psi_{a}^{h}\right|^{2}$ from $I_{1}$. In particular, they are related to each other by the relation,

$$
X_{h}=\sqrt{I_{1, h}}=b(t) X(\tau(t)),
$$

where $b(t)$ and $\tau(t)$ are determined from the Eq. (15). Thus, even though the exact solution of the equation of motion of $\mathcal{A}_{h}$ is not known, the dynamics of $X_{h}$ can be described exactly.

An alternative, but, useful expression for the $X_{h}$ can be determined from the following equation [3],

$$
\frac{d^{2} X_{h}}{d t^{2}}+\omega(t) X_{h}=\frac{Q_{h}}{X_{h}^{3}}, Q_{h}=I_{1, h} H_{h}-I_{2, h}^{2},
$$

where $Q_{h}$ is a constant of motion. Both $H_{h}$ and $I_{2, h}$ have the same expressions as in $H$ and $I_{2}$, respectively, with $\left(\tau, \mathbf{r}, \psi_{a}\right)$ replaced by $\left(t, \mathbf{r}_{h}, \psi_{a}^{h}\right)$. Note that the Eq. (17) can also be interpreted as describing the motion of a classical particle in a combined harmonic and inverse-square potential. The particle falls to the center for an attractive $\left(Q_{h}<0\right)$ inverse-square potential, independent of the time-dependence of the harmonic trap. This implies that the condensate collapses at a finite time for $Q_{h}<0$. Analyzing the exact expression for $Q_{h}$ further, we find that the condensate collapses for any initial condition if $H_{h} \leq 0$. For $H_{h}>0$, the condition for the collapse is given by,

$$
\left.\frac{d I_{1, h}}{d t}\right|_{t=0}<-2 \sqrt{\left.\left(I_{1, h} H_{h}\right)\right|_{t=0}},
$$

where the relation [3], $\dot{I}_{1, h}=2 I_{2, h}$, valid for the system described by the action $\mathcal{A}_{h}$ has been used. As far as we are aware of, this is for the first time in the literature that a criteria for the collapse of the condensate in the most general two dimensional multi-component GPE with cubic nonlinearity and an arbitrary time-dependent harmonic trap is given. Note that the criteria is independent of the total number of components $n$ and any additional internal global symmetry. The known results for the single-component 26] and the multi-component 117] GPE with time-independent harmonic trap in $2+1$ dimensions are easily reproduced from this very general result. Further, the criteria for the collapse in the system without or with the harmonic trap is also identical, except that there is no equality sign in (18) for the later case [ compare with Eq. (12)]. This is precisely because, for $Q, Q_{h}=0$, Eqs. (9) and (17) describe the dynamics of a free particle and that of a particle in a time-dependent 
harmonic trap, respectively. Thus, nothing can be said conclusively on the dynamical (in)stability for the later case, unless the time-dependence of the frequency of the trap is explicitly specified. For a time-independent trap, the equality sign is recovered in Eq. (18); and of course, the known result 17,26] is identically reproduced.

We have shown that the criteria for the collapse of the condensate in a $2+1$ dimensional system governed by the Lagrangian $\mathcal{L}_{h}$ is independent of the total number of components $n$. It is known [26] that the same criteria for the collapse of the condensate is also valid for the Lagrangian $\mathcal{L}_{h}$ in dimensions $d \geq 2+1$ with $n=1$, $\omega(t)=\omega_{0}=$ a constant and $\dot{f}=0$. So, the criteria for the collapse is independent of the underlying $O(2,1)$ symmetry, which the cubic NLSE has only in $d=2+$ 1. The dynamical $O(2,1)$ symmetry only helps us in deriving the exact result in a much more simpler and elegant way. Based on this observation, we conjecture that the criteria for the collapse of the condensate of $\mathcal{L}_{h}$ in dimensions $d \geq 2+1$, with $\omega(t)=\omega_{0}$ and $\dot{f}=0$, is independent of the total number of components $n$ and the criteria is the same as stated in this note for $d=2+1$. Note that the physically interesting case of $d=3+1$ is also included in our conjecture.

The solution for $X_{h}$ is given by,

$$
X_{h}^{2}=u^{2}(t)+\frac{Q_{h}}{W^{2}} v^{2}(t), W(t)=u \dot{v}-v \dot{u},
$$

where $u(t)$ and $v(t)$ are two independent solutions of Eq. (15) satisfying $u\left(t_{0}\right)=X_{h}\left(t_{0}\right), \dot{u}\left(t_{0}\right)=\dot{X}_{h}\left(t_{0}\right), \dot{v}\left(t_{0}\right)=0$, and $v\left(t_{0}\right) \neq 0$. The above solution is valid for arbitrary $Q_{h}$ : positive, negative or zero. We will be considering the case $Q_{h} \geq 0$ from now onwards, since we have already argued that the condensate collapses for $Q_{h}<0$. We have obtained the same expressions (16) and (19) in Refs. [3, 1 for the dynamics of the width of the wavepacket of a single-component condensate in $2+1$ dimensions. So, the results of the Refs. [3, [4 are equally valid for the general multi-component condensate in $2+1$ dimensions with the moment $I_{1}=X_{h}^{2}$ identified as the total mean-square radius. In particular,

(a) the system described by $\mathcal{L}_{h}$ has an explosionimplosion duality for $\dot{f}(t)=0$ and either $\omega(t)=0$ or $\omega(t)=t^{-2}$

(b) the condensate exhibits extended parametric resonance for a periodic $\omega(t)$ and arbitrary $f(t)$,

(c) the dynamic (in)stability of the system is independent of $f(t)$, i.e., same for both rotating as well as non-rotating BEC.

We refer the readers to Refs. [3, 3 ] for further details.

Finally, we conclude with the following comment. The results presented in this note for the multi-component BEC are a generalization of what already has been known for the single-component BEC in two dimensions. The results obtained in both these cases are also identical with the identification of the moment $I_{1}$ as the total mean square radius. In particular, the dynamics of the moment $I_{1}$ is determined from the same solvable equation as in the case of a single-component BEC with all the information about the Hamiltonian encoded into the constant of motion $Q_{h}$. Apart from its relevance to the ongoing experiments on BEC, the importance of these results lie in its universality. No matter how many components are there, or how they interact among themselves, or even whether they are rotating or non-rotating, the dynamics of the total mean-square radius is universally determined by the same equation. This is indeed a counter-intuitive result and may be realized in the laboratory in near future.

\section{ACKNOWLEDGMENTS}

I would like to thank T. Deguchi and T. K. Ghosh for a careful reading of the manuscript and comments. I would like to thank J. Ieda, H. Morise, N. Uesugi, M. Wadati and especially T. Tsurumi for useful discussions. This work is supported by JSPS.

[1] F. Dalfovo, S. Giorgini, L. P. Pitaevskii and S. Stringari, Rev. Mod. Phys. 71463 (1999) (cond-mat/9806038).

[2] We refer to the papers in Refs. 118 and 19 for the application of $O(2,1)$ symmetry in physics. The models considered in these papers also have a dynamical $O(2,1)$ symmetry.

[3] P. K. Ghosh, Phys. Rev. A65, 012103 (2002) ( condmat/0102488).

[4] P. K. Ghosh, cond-mat/0109073.

[5] J. J. Garcia-Ripoll and V. M. Perez-Garcia, Phys. Rev. Lett. 83, 1715 (1999); patt-sol/9904006.

6] T. K. Ghosh, Phys. Lett. A 285, 222 (2001 ) ( condmat/0012188).

[7] I. O'C Drury and J. T. Mendonca, Physics of Plasmas 7, 5148 (2000) (astro-ph/0003385).

[8] K. Walter, Supernova Hydrodynamics Up Close: Science and Technology Review, Jan'/Feb' 2000 ( www.llnl.gov/str/BRemington.html); I. Hachisu et. al., Astrophysical Journal 368, L27 (1991); H. Sakagami and K. Nishihara, Physics of Fluids B 2, 2715 (1990).

[9] J. Stenger, S. Inouye, D. M. Stamper-Kurn, H.-J. Miesner, A. P. Chikkatur and W. Ketterle, Nature 369, 345 (1998); H.-J. Miesner, D. M. Stamper-Kurn, J. Stenger, S. Inouye, A. P. Chikkatur and W. Ketterle, Phys. Rev. Lett. 82, 2228 (1999); D. M. Stamper-Kurn, H.-J. Miesner, A. P. Chikkatur, S. Inouye, J. Stenger and W. Ketterle, Phys. Rev. Lett. 83, 661 (1999).

[10] C. J. Myatt, E. A. Burt, R. W. Ghrist, E. A. Cornell and C. E. Wieman, Phys. Rev. Lett. 78, 586 (1997); M. R. Matthews, D. S. Hall, D. S. Jin, J. R. Ensher, C. E. Wieman, E. A. Cornell, F. Dalfovo, C. Minniti and S. 
Stringari, Phys. Rev. Lett. 81, 243 (1998); D. S. Hall, M. R. Matthews, J. R. Ensher, C. E. Wieman and E. A. Cornell, Phys. Rev. Lett. 81, 1539 (1998).

[11] T. Ohmi and K. Machida, J. Phys. Soc. Jpn. 67, 1822 (1998).

[12] T.-L. Ho, Phys. Rev. Lett. 81, 742 (1998).

[13] D. M. Stamper-Kurn and W. Ketterle, cond-mat/0005001.

[14] A. J. Leggett, Rev. Mod. Phys. 73, 307 (2001).

[15] T.-L. Ho and V. B. Shenoy, Phys. Rev. Lett. 77, 3276 (1996); B. D. Esry, C. H. Greene, J. P. Burke, Jr. and J. L. Bohn, Phys. Rev. Lett. 78, 3594 (1997).

[16] S.-K. Yip, Phys. Rev. Lett. 83, 4677 (1999); U. Leonhardt and G. E. Volovik, JETP Lett. 72, 46 (2000); J. Ruostekoski and J. R. Anglin, Phys. Rev. Lett. 86, 3934 (2001); H. T. C. Stoof, E. Vliegen and U. Al Khawaja, Phys. Rev. Lett. 87, 120407 (2001); U. Al Khawaja and H. Stoof, Nature 411, 918 (2001); K.-P. Marzlin, W. Zhang and B. C. Sanders, Phys. Rev. A 62, 013602 (2000).

[17] T. Tsurumi and M. Wadati, J. Phys. Soc. Japan 67, 93 (1998).

[18] V. de Alfaro, S. Fubini and G. Furlan, Nuvo Cimento A34, 569 (1976).

[19] R. Jackiw, Ann. Phys. (N.Y.) 129, 183(1980); 201, 83 (1990).

[20] C. R. Hagen, Phys. Rev. D5, 377 (1972).
[21] U. Niederer, Helv. Phys. Acta 45, 802 (1972); 46, 191 (1973); 47, 119, 167 (1974); 51, 220 (1978).

[22] E. A. Kuznetsov and S. K. Turitsyn, Phys. Lett. A112, 273 (1985).

[23] S. Takagi, Prog. Theor. Phys. 84, 1019 (1990); 85, 463 (1991); 85, 723 (1991).

[24] Pijush K. Ghosh, Phys. Rev. D 53, 2248 (1996) ( hepth/9503199 ).

[25] V. E. Zakharov, Sov. Phys. JETP 35, 908 (1972); V. E. Zakharov and V. S. Synakh, Sov. Phys. JETP 41, 465 (1975); M. I. Weinstein, Commun. Math. Phys. 87, 567 (1983).

[26] T. Tsurumi and M. Wadati, J. Phys. Soc. Japan 66, 3031 (1997); ibid 66, 3035 (1997); M. Wadati and T. Tsurumi, Phys. Lett. A 247, 287 (1998); T. Tsurumi, H. Morise and M. Wadati, Int. Jour. Mod. Phys. B 14, 655 (2000) ( cond-mat/9912470).

[27] A. V. Rybin, G. G. Varzugin, M. Lindberg, J. Timonen and R. K. Bullough, Phys. Rev. E 62, 6224 (2000) ( cond-mat/0001059 ).

[28] S. Stringari, Phys. Rev. Lett. 77, 2360 (1996); V. M. Perez-Garcia et. al., Phys. Rev. Lett. 77, 5320 (1996).

[29] S. N. Vlasov, V. A. Petrischev and V. I. Talanov, Radiophys. Quantum Electron. 14, 1062 (1971); V. M. PerezGarcia et. al., J. Opt. B : Quantum Semiclass. Opt. 2, 353358 (2000). 